Z. X. Chen, N. Swaminathan, M. Stöhr and W. Meier, Interaction between self-excited oscillations and fuel-air mixing in a dual swirl combustor, Proceedings of the Combustion Institute 37 (2019) $2325-2333$.

The original publication is available at www.elsevier.com

https://doi.org/10.1016/j.proci.2018.08.042 


\title{
Interaction between self-excited oscillations and fuel-air mixing in a dual swirl combustor
}

\author{
Zhi X. Chen ${ }^{\mathrm{a}, *}$, Nedunchezhian Swaminathan ${ }^{\mathrm{a}}$, Michael Stöhr ${ }^{\mathrm{b}}$, Wolfgang Meier $^{\mathrm{b}}$ \\ ${ }^{a}$ Department of Engineering, University of Cambridge, Cambridge CB2 1PZ, UK \\ ${ }^{b}$ Institute of Combustion Technology, German Aerospace Center (DLR), Pfaffenwaldring 38-40, Stuttgart 70569, Germany
}

\begin{abstract}
A partially premixed gas turbine model combustor close to an industrial design is investigated using Large Eddy Simulation (LES). Two flames, one stable and another unstable with self-excited oscillations are computed. In particular, this study addresses the previously unexplained transition of flame shape in the experiments, from V-shaped to flat when the flame becomes acoustically unstable, suggesting a notable change of the important convective delay in the thermoacoustic feedback loop. The LES results show good agreement with the measured velocities, temperature and mass fractions. The acoustic power spectral density (PSD) obtained from the LES of the unstable flame also agrees well with the measured amplitudes in the air plenum and combustion chamber, and reasonably captures the frequency with a slight under-prediction. A comparison of the stable and unstable cases shows different mixing and reaction behaviours despite similar mean velocity fields. Further detailed analysis shows that the different mixing behaviour is driven by the significantly varying air mass split between the two air passages during a thermoacoustic oscillation cycle. This variation is due to the different impedances experienced by the pressure oscillations propagating through the two swirling injector passages with different internal geometries. This causes a periodic variation of the radial momentum of the fuel jets injected between the two swirling air flows. The resulting flapping of the fuel jets creates an enhanced radial fuel-air mixing that leads to a flattened flame in the unstable case. This provides a new physical explanation for the transitions of flame shape observed in the experiments.
\end{abstract}

Keywords: Large Eddy Simulation, Thermoacoustic instability, Self-excited, Dual swirl, Partially premixed flame

\section{Introduction}

Thermoacoustic instability is an inevitable issue for gas turbines (GT) operating at lean conditions [1,2]. This phenomenon is driven by the interaction among the fluctuations of turbulent flow, mixing and heat release rate and acoustic pressure. If a positive feedback loop is established, this results in a growth of oscillation amplitude leading to a limit cycle [2,3]. Hence, thermoacoustic instability has been studied in the past using analytical [4-6] and simple numerical $[7,8]$ techniques, which typically decouples reacting flow and linearised system acoustics. In these low-order methods, the flame is seen as compact sources [7] for pressure perturbation and is typically described using flame transfer or describing functions (FTF or FDF) for acoustic analysis. Many past experimental and numerical works attempted

\footnotetext{
* Corresponding author:

Email address: zc252@cam.ac.uk (Zhi X. Chen)
}

to provide the FTF or FDF by studying the flame response to velocity or pressure perturbations excited by external forcing to investigate longitudinal instabilities (see [9] for a review). Due to the complexity of the feedback mechanisms, however, the predictive capability of this approach remains limited such that experimental tests of FTF/FDF often must be repeated for modified combustor designs.

The compressible Large Eddy Simulation (LES) is becoming a promising tool to predict self-excited instabilities since the unsteady heat release and its coupling with the acoustics are intrinsically dealt with by the LES equations and sub-models without assuming a compact flame and acoustic linearity. Past LES studies of self-induced oscillations in laboratory [10-12] and practical $[13,14]$ combustors provided insights into the physical mechanism of these oscillations [3].

An important basis for development of advanced LES is the availability of experimental validation data. Several comprehensive data sets have been re- 
ported for a number of gas turbine model combustors (GTMCs) [15]. One well-established GTMC is the DLR dual swirl burner investigated by Meier et al. [1618], who acquired extensive datasets for both acoustically stable and unstable flames. One interesting phenomenon seen in their studies was that acoustically stable flames generally exhibit an elongated V-shaped form, whereas the shape changes to a flattened form along the base plate when the flame becomes acoustically unstable. The mechanisms causing this effect, which implies a notable change of the important convective delay in the thermoacoustic feedback loop, remained unclear.

Until now only the stable flame of the DLR-GTMC was attempted using LES [19-21]. The objective here is to perform compressible LES of this dual swirl GTMC with self-excited oscillations and to offer physical insights for the flow/flame structures and their interactions. The specific focus is to investigate the changes in flame shape using the simulation data of the stable and unstable flames, and to provide a physical explanation for this phenomenon. The remainder of this paper is organised as follows. The LES modelling framework is described in Section 2, followed by the experimental and numerical details in Section 3. The results are discussed in Section 4 and the conclusions are summarised in Section 5.

\section{Modelling methodology}

The compressible Favre-filtered conservation equations for mass and momentum are solved. The subgrid scale (SGS) stresses are modelled using the nondynamic Smagorinsky model. The flamelet model used for this study maps all thermo-chemical quantities into mixture fraction, $Z$, and a reaction progress variable, $c$, spaces and utilises a presumed joint SGS probability density function (PDF) for $Z$ and $c$. The mixture fraction is defined using Bilger's formula [3]. The normalised progress variable is used: $c=\psi / \psi^{\mathrm{Eq}}$, where $\psi=Y_{\mathrm{CO}}+Y_{\mathrm{CO} 2}$ and $\psi^{\mathrm{Eq}}$ is its equilibrium value for the local mixture [22]. The first two moments of $Z$ and $c$, filtered means and SGS variances, along with the thermochemical enthalpy are solved using their transport equations:

$$
\bar{\rho} \frac{D \widetilde{\varphi}}{D t}=\nabla \cdot\left[\left(\bar{\mu}+\frac{\mu_{t}}{\mathrm{Sc}_{t}}\right) \nabla \widetilde{\varphi}\right]+\overline{S_{\varphi}^{+}}-\overline{S_{\varphi}^{-}},
$$

where the vectors of transported Favre-filtered scalars, source and sink terms are respectively given as

$$
\begin{aligned}
\widetilde{\varphi}= & \left\{\widetilde{Z}, \widetilde{Z^{\prime \prime 2}}, \widetilde{c}, \widetilde{c^{\prime \prime 2}}, \widetilde{h}\right\}, \\
\overline{\boldsymbol{S}_{\varphi}^{+}}= & \left\{0,2 \frac{\mu_{t}}{\mathrm{Sc}_{t}}|\nabla \widetilde{Z}|^{2}, \overline{\dot{\omega}_{c}^{*}},\right. \\
& \left.2 \frac{\mu_{t}}{\mathrm{Sc}_{t}}|\nabla \widetilde{c}|^{2}+2\left(\overline{c \dot{\omega}_{c}^{*}}-\widetilde{c} \overline{\dot{\omega}_{c}^{*}}\right), \frac{D \bar{p}}{D t}\right\}, \\
\overline{\boldsymbol{S}_{\varphi}^{-}}= & \left\{0,2 \bar{\rho} \widetilde{\chi}_{Z, \mathrm{sgs}}, 0,2 \bar{\rho} \widetilde{\chi}_{c, \mathrm{sgs}}, 0\right\} .
\end{aligned}
$$

The filtered molecular and turbulent eddy dynamic viscosities are $\bar{\mu}$ and $\mu_{t}$ respectively. A turbulent Schmidt number of $\mathrm{Sc}_{t}=0.4$ is used for all scalars [22] and $\bar{p}$ is the filtered pressure. The remaining unclosed sources and sinks in Eqs. (3)-(4) are the reaction source terms, $\overline{\dot{\omega}_{c}^{*}}$ and $\overline{c \dot{\omega}_{c}^{*}}$, and the SGS scalar dissipation rates, $\widetilde{\chi}_{Z \text {, sgs }}$ and $\widetilde{\chi}_{c \text {, sgs. }}$ Their closure models are described next.

As the combustion is partially premixed in this study, a subgrid model accounting for both premixed and nonpremixed combustion modes is used and the filtered reaction rate is: $\overline{\dot{\omega}}_{c}^{*}=\overline{\dot{\omega}}_{c}+\overline{\dot{\omega}}_{\text {np }}$ [22]. The premixed term is modelled as

$\overline{\dot{\omega}}_{c}=\bar{\rho} \int_{0}^{1} \int_{0}^{1} \frac{\dot{\omega}_{c}(\xi, \zeta)}{\rho(\xi, \zeta)} \widetilde{P}(\xi, \zeta) d \zeta d \xi$,

where $\xi$ and $\zeta$ are the sample space variables for $Z$ and $c$ respectively. The flamelet reaction rate, $\dot{\omega}_{c}$, and density, $\rho$, are computed using planar freely-propagating laminar flames for different mixture fractions covering the flammable range. The SGS joint PDF is approximated as $\widetilde{P}(\xi, \zeta) \approx \widetilde{P}_{\beta}\left(\xi ; \widetilde{Z}, \widetilde{Z^{\prime \prime 2}}\right) \times \widetilde{P}_{\beta}\left(\zeta ; \widetilde{c}, \widetilde{c^{\prime \prime 2}}\right)$ using a presumed $\beta$-distribution for both marginal PDFs. For the non-premixed term, an algebraic model is used [22]:

$\overline{\dot{\omega}}_{\mathrm{np}} \simeq \widetilde{c}\left(\bar{\mu}|\nabla \widetilde{Z}|^{2}+\bar{\rho} \widetilde{\chi}_{Z, \mathrm{sgs}}\right) \int_{0}^{1} \frac{1}{\psi^{\mathrm{Eq}}} \frac{d^{2} \psi^{\mathrm{Eq}}}{d \xi^{2}} \widetilde{P}_{\beta}(\xi) d \xi$,

where the SGS scalar dissipation rate $\widetilde{\chi}_{Z \text {, sgs }}$ is modelled using a linear relaxation approximation [3]. For the progress variable dissipation rate, $\widetilde{\chi}_{c \text {, sgs }}$, a well validated model used in [22] is adopted.

The reaction term, $\overline{c \dot{\omega}_{c}^{*}}$, in Eq. (3) and other thermochemical quantities are calculated in a similar manner as Eq. (5) and the filtered mixture density is computed using the ideal gas state equation: $\bar{\rho}=\bar{p} /\left(\widetilde{T} \mathscr{R}_{0} / \widetilde{W}_{\text {mix }}\right)$, where $\widetilde{T}$ and $\widetilde{W}_{\text {mix }}$ are the mixture temperature and molecular weight, respectively. The universal gas constant is $\mathscr{R}_{0}=8.314 \mathrm{~J} / \mathrm{mol} / \mathrm{K}$. A lookup table containing various sources and thermo-chemical properties is constructed with $\widetilde{Z}, \widetilde{Z^{\prime \prime 2}}, \widetilde{c}$ and $\widetilde{c^{\prime \prime 2}}$ as control vari- 
ables, and the values required for LES are obtained using linear interpolation with an error less than $1 \%$ [23]. This modelling framework has been extensively validated for Reynolds-Averaged Navier-Stokes (RANS) paradigm using various configurations [23-25]. It was recently assessed for LES in [22] showing good predictions for transiently igniting jet flames and here is further validated using the DLR dual swirl burner which is described next.

\section{Experimental case and numerical setup}

Figure 1 shows a schematic of experimental configuration of the burner arrangement $[16,17]$. The injector comprises two nozzles for swirled air (co-rotating) with an annular ring of fuel jet nozzles in-between. The exit of the inner swirler and fuel jets is $4.5 \mathrm{~mm}$ below the outer swirler exit which is set as $h=0$ in the axial direction. Dry ambient air was supplied through the air feed pipe and non-swirling methane was supplied through 72 square channels $(0.5 \times 0.5 \mathrm{~mm})$ and these small squares are retained in the numerical grid to capture the correct mixing. In this study, one non-reacting and two reacting flow cases are simulated, see Table 1 for details. The inlet mass flow rates for air plenum and fuel jet nozzle are denoted by $\dot{m}_{\mathrm{p}}$ and $\dot{m}_{\mathrm{j}}$ respectively. The swirl number, $S$, is defined using a standard formula in [16].

Table 1. Summary of the simulated cases.

\begin{tabular}{lllll}
\hline Case & $\Phi_{\text {glob }}$ & $\dot{m}_{\mathrm{p}}(\mathrm{g} / \mathrm{s})$ & $\dot{m}_{\mathrm{j}}(\mathrm{g} / \mathrm{s})$ & $S$ \\
\hline Non-reacting & - & 19.74 & 1.256 & 0.9 \\
F-A (stable) & 0.65 & 18.25 & 0.697 & 0.9 \\
F-B (unstable) & 0.75 & 4.68 & 0.205 & 0.55 \\
\hline
\end{tabular}

Numerical boundary conditions, inlet and outlet impedances in particular, are important for LES of thermoacoustics because they significantly affect the selfexcitation process [3] . However, it is not straightforward to specify these impedances for the combustion chamber and thus the full geometry including both the upstream and downstream of the combustion chamber is included as in Fig. 1b. The mass flow rates in Table 1 at the fuel and air inlets, which are fully reflective, are specified [12]. Sensitivity of the far-field outlet boundary location from the combustor exhaust duct exit (see Fig. 1) was studied for LES . It was found that a distance of the order of typical acoustic wavelength in ambient air $(\sim 1.3 \mathrm{~m})$ at the oscillation frequency of about $250 \mathrm{~Hz}$ is required to avoid acoustic wave reflection from the outlet. No-slip wall conditions are employed for combustor walls. An unstructured grid is used for which more details are given later in subsection 4.1. OpenFOAM 2.3.0 with schemes of second-order accuracy in time and space is used. The time-step size is determined to have a maximum acoustic $\mathrm{CFL}<0.5$. After passing the transients, LES statistics are collected over 12 flowthrough-time which is about 0.06 and $0.2 \mathrm{~s}$ for flames F-A and F-B, respectively. The simulations are performed on the ARCHER UK National Supercomputer using 1080 cores and computing one flow-through-time for both flames requires about 12 hours on a wall-clock.

\section{Results and Discussion}

\subsection{Non-reacting flow and mesh sensitivity}

Measured mean and r.m.s. axial velocities [26] are compared with the LES results in Fig. 2. Three sets of LES results are shown for numerical grids designated as G1, G2 and G3, having 12, 15 and 20 million cells, with typical sizes of $1.2,0.5$ and $0.3 \mathrm{~mm}$, respectively. The grid cells having a size of $0.1 \mathrm{~mm}$ are uniformly spaced inside the fuel inlets for all three cases. A good overall agreement is seen for G2 with significant improvement from G1 and small difference is found from G3. It can be seen that both the mean and r.m.s. are well captured in the near-field $(h=2.5 \mathrm{~mm})$ suggesting a good prediction of the flow inside the swirlers using G2 and G3. However, for the coarse grid G1 the peak value is under-predicted and its radial location further away from the centre, i.e., $x=0 \mathrm{~mm}$. This becomes more evident in the downstream where the inner recirculation zone (IRZ) width is over-estimated by about $80 \%$ at $h=20 \mathrm{~mm}$ for G1. This IRZ width is predicted quite well for G2 and G3, and thus grid G2 is chosen for the subsequent reacting flow simulations.

\subsection{Reacting flow/flame structures: stable vs. unstable}

Figure 3 compares the averaged mid-plane $\mathrm{CH}$ contours using the computed mass fraction from the LES and planar laser-induced fluorescence (PLIF) measurements [16]. High $\mathrm{CH}$ concentration is used here as a qualitative marker of the reaction zone [17]. It is seen in Fig. 3a that the stable flame F-A shows a conical structure of typical swirl flames and the reaction zone is mainly located in the shear layer between the IRZ and main inflow stream. The overall shape of $\mathrm{CH}$ distribution in F-A is captured quite well although the spreading angle from the centreline is slightly over-predicted. This is a consequence of the difficulties in capturing the separation point on the contoured outer air nozzle. In contrast, the flame F-B exhibits a different shape as in Fig. 3b, flattened towards the combustion chamber 

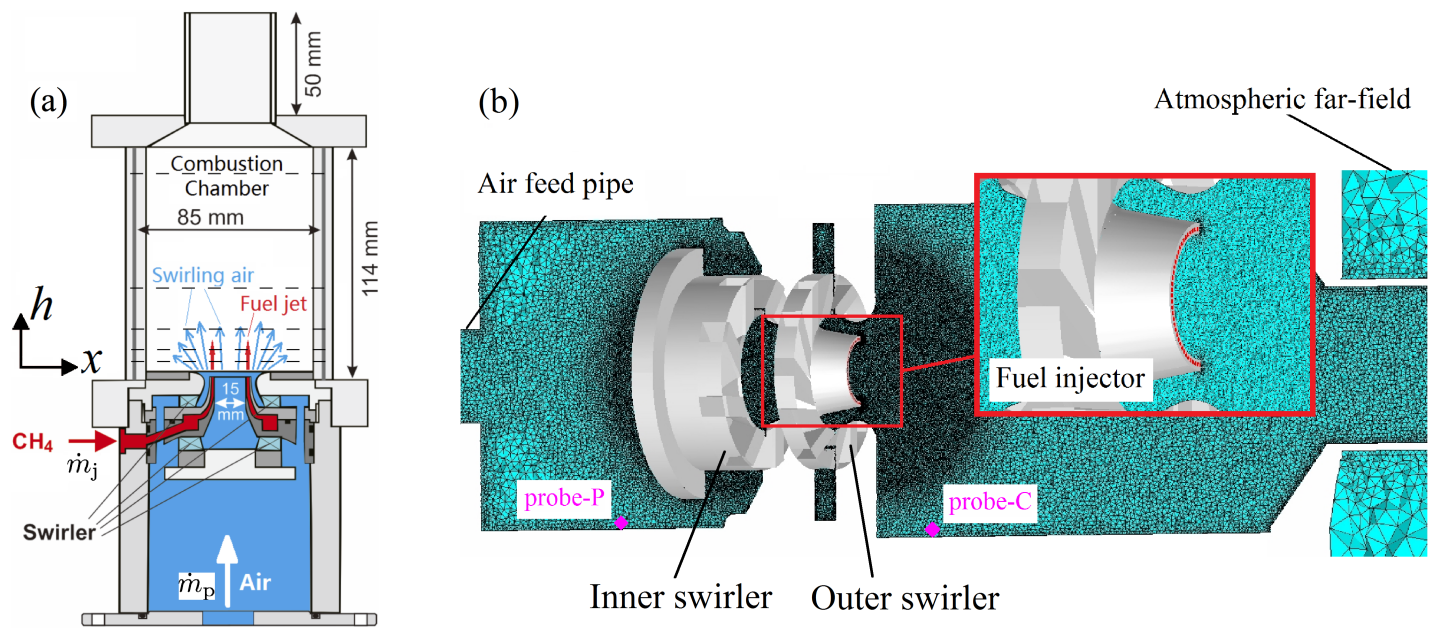

Fig. 1. Schematic of the (a) dual swirl burner [16, 17], (b) numerical grid on mid-plane.

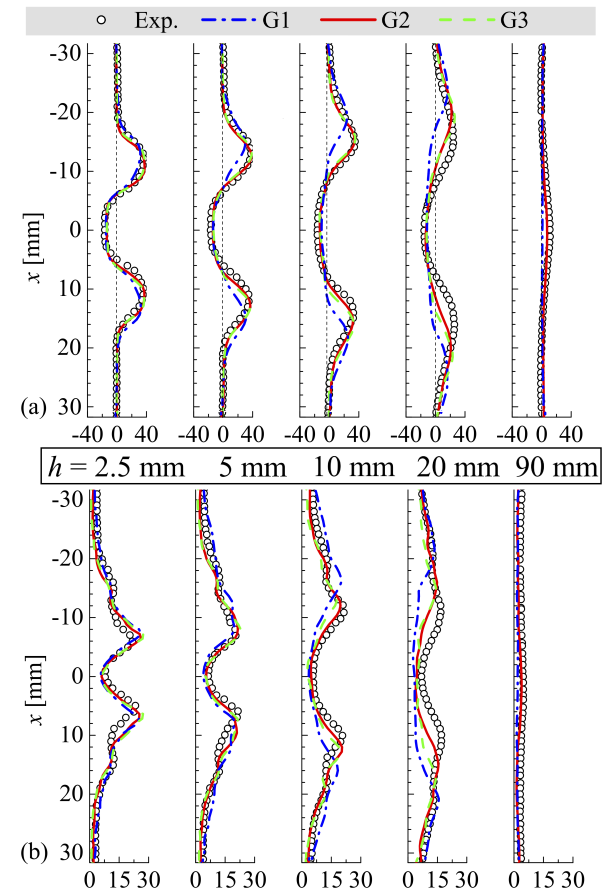

Fig. 2. Cold flow comparison of (a) mean (b) r.m.s. axial velocities: measurements [26] (symbols) and LES (lines) with three grids.

bottom. The LES seems to capture this change in the flame shape quite well. The mean flat flame arises because of thermoacoustic oscillations leading to a periodic variation in the air flow and a radial swaying of the fuel jet, which is further discussed in subsection 4.3. A plot (not shown) of the Rayleigh criterion, $\int_{V} p^{\prime} q^{\prime} d V$, where $p^{\prime}$ and $q^{\prime}$ are fluctuating pressure and heat re-

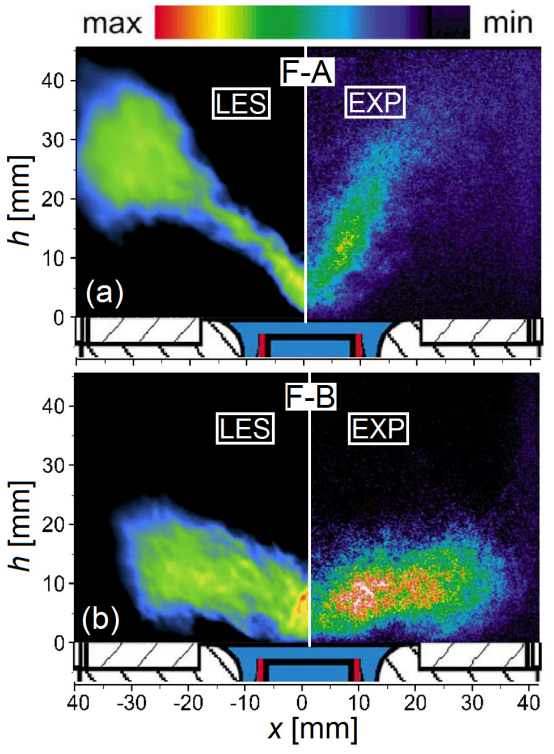

Fig. 3. Mid-plane time-averaged $\mathrm{CH}$ fields obtained from LES and measurements [16] for flames (a) F-A and (b) F-B.

lease rate, clearly identifies that this criterion is predominantly positive for the flame F-B and negative for F-A as one would expect. The flame lift-off height, defined as the axial distance between the fuel jet exit plane and the base of the central reaction zone, are computed to be about 4 and $3.5 \mathrm{~mm}$ for the flames F-A and F-B respectively, which agree well with the measurements of about 5 and $4 \mathrm{~mm}$ [16]. Computed axial and radial velocities are compared against the measurements [16] in Fig. 4 for both flames. Both the mean and r.m.s. values are presented for few typical axial locations cover- 
(a) F-A: Averaged velocities $[\mathrm{m} / \mathrm{s}]$
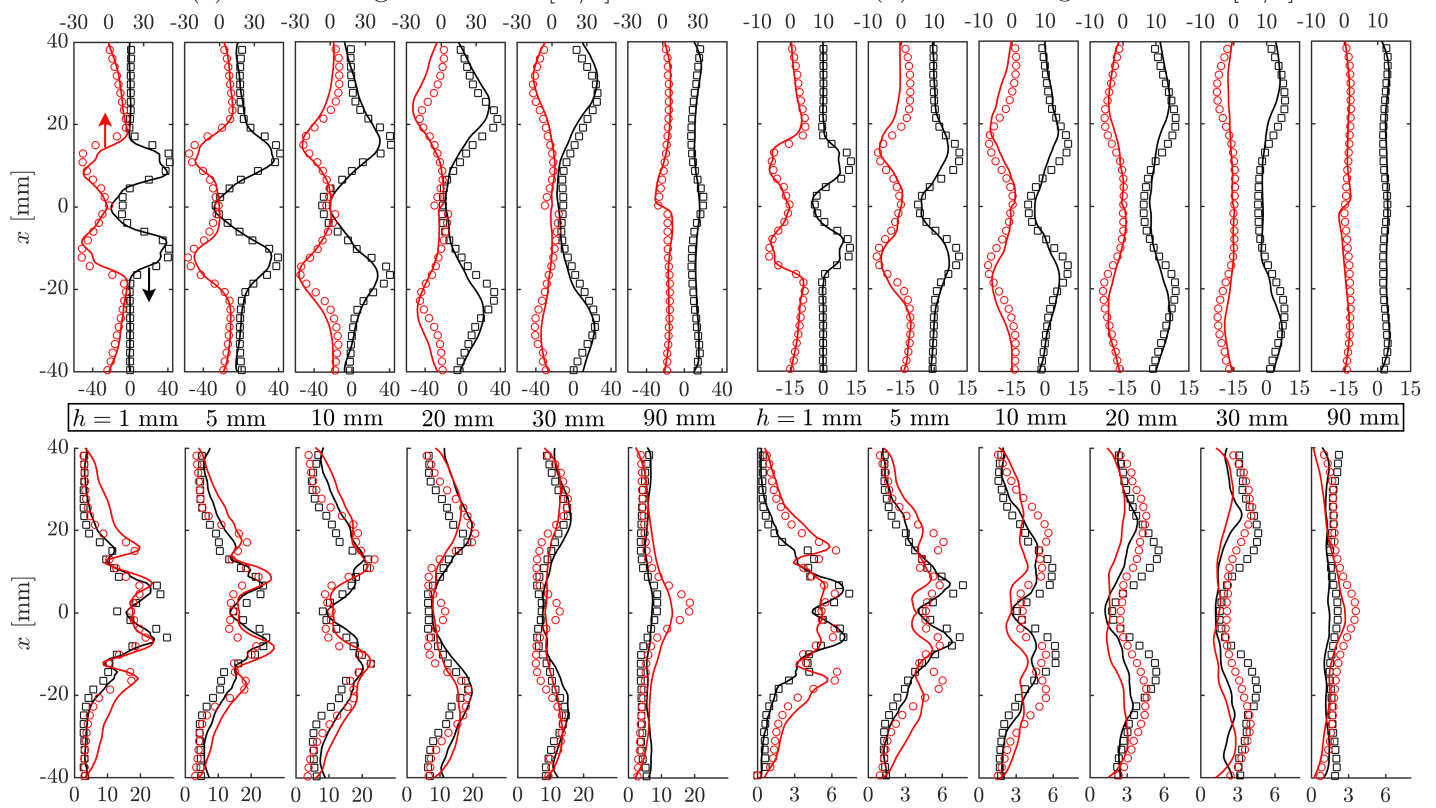

(c) F-A: r.m.s. velocities $[\mathrm{m} / \mathrm{s}]$

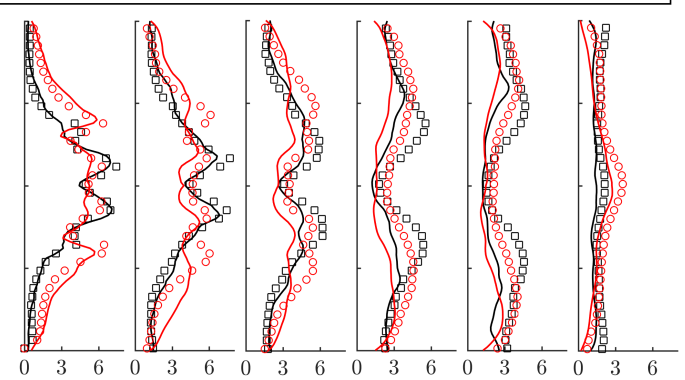

(d) F-B: r.m.s. velocities $[\mathrm{m} / \mathrm{s}]$

Fig. 4. Typical comparison of axial (black) and radial (red) velocities: measurements [16, 17] (symbols) and LES (lines).

ing the entire combustion chamber ( $h=1$ to $90 \mathrm{~mm}$ ). Overall, a good agreement is seen for both velocities in the two flames. Similar agreement is also observed for azimuthal component (not shown). Some underprediction is observed in the peak mean axial velocity at $h=20$ and $30 \mathrm{~mm}$ in Figs. $4 \mathrm{a}$ and $4 \mathrm{~b}$. This is the cause for the over-predicted flame spreading angle in the F-A case in Fig. 3a leading to some over-prediction of radial velocity at these locations. The pattern of velocity variations is quite similar for both flames as shown in Fig. 4. However, the magnitude of the velocity components are different as indicated by the $x$-axis values in the figure. Although the velocity patterns are similar, the flame shapes are different. The flame shape in the FA case mainly depends on the mean flow structure with the reaction mostly occurring in the inner shear layer, whereas the flame in the F-B case is significantly influenced by the periodic thermoacoustic oscillations. This fluctuating pressure causes periodic changes in the air flow rates through the inner and outer swirlers. As a result, the transient flow structures modify the fuel-air mixing patterns leading to different reaction zone shape and distribution as seen in Fig. 3.

Figure 5 compares the measured [16] and computed averaged temperature, $\langle\widetilde{T}\rangle$, and fuel mass fraction, $\left\langle\widetilde{Y}_{\mathrm{CH} 4}\right\rangle$ for the flames F-A and F-B, and the comparison is quite good for both flames. The over-estimation
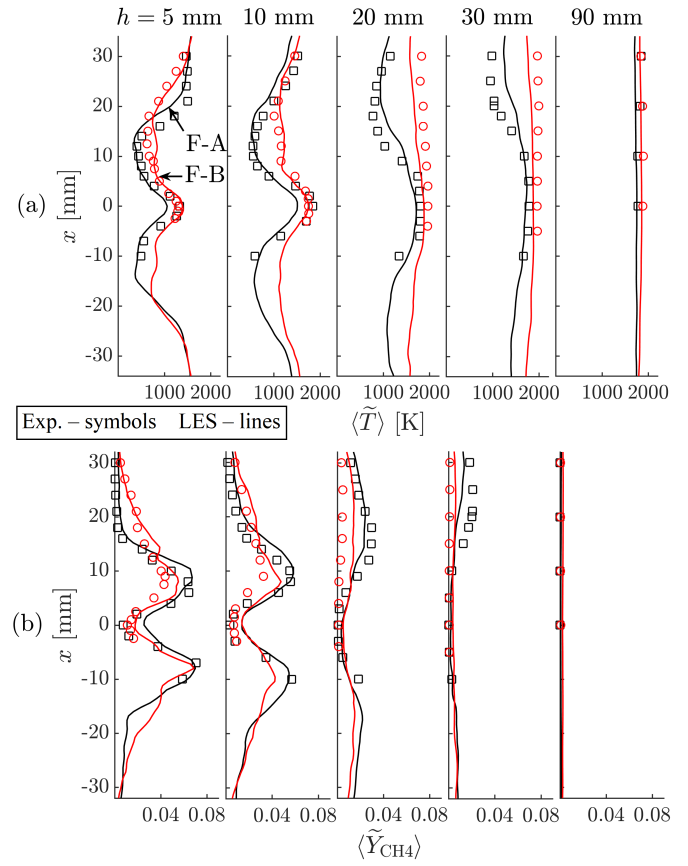

Fig. 5. Typical comparison of averaged temperature and fuel mass fraction.

of $\langle\widetilde{T}\rangle$ at $h=20$ and $30 \mathrm{~mm}$ for the F-A is caused by the smaller axial penetration of fresh gases noted in Fig. 4. 
Although similar behaviour is observed for the F-B, the mean temperature is well predicted since the mixture is almost fully burnt with $\langle\widetilde{T}\rangle \approx 2000 \mathrm{~K}$, and therefore it is less influenced by the velocity field. An interesting point noted in $[16,17]$ is that a substantial amount of fuel leaks into the outer region $(|x|>15 \mathrm{~mm})$ at $h=5 \mathrm{~mm}$ in the F-B case whereas the fuel mass fraction is nearly zero in this region for the F-A flame. This behaviour is also observed in the LES as in Fig. 5b and is likely to be correlated with the thermoacoustic oscillations causing the flame to flatten. However, the driving mechanism behind this flame flattening was unclear from the measurements and thus it is investigated using the LES data in the next subsection.

\subsection{Thermoacoustic instability and its interaction with upstream fuel-air mixing}

The acoustic behaviour exhibited in the flame F-B is examined in Fig. 6 by comparing the measured and computed pressure fluctuation, $p^{\prime}$, and its power spectral density (PSD) for two probes $\mathrm{P}$ and $\mathrm{C}$ located respectively in the air plenum and combustion chamber. The time series of $p^{\prime}$ for $60 \mathrm{~ms}$ is arbitrarily chosen from the experimental and LES data. It is seen that the computed pressure signals compare quite well with the measurements for both probes. The PSDs presented in Fig. 6 are computed using $p^{\prime}$ time series for $1 \mathrm{~s}$ and $0.15 \mathrm{~s}$ obtained from the experiment and LES having a spectral resolution of 1 and $6.7 \mathrm{~Hz}$ respectively. The oscillation frequency is captured quite well in the LES with an under-prediction of $34 \mathrm{~Hz}$ at both probes. This difference may be attributed to the absence of the air supply pipe (length of several metres) in the LES as suggested by recent experiment [27] using a choked air inflow. Another possible reason is that the plenum which supplies methane to the fuel nozzles can also play as a resonator for the oscillations. A numerical study [12] showed improved results with the fuel plenum included for the PRECCINSTA burner and this effect on the dual swirl burner will be explored in a future work. The computed peak PSD agrees quite well with the measured values and the LES is also able to reproduce the subharmonic and higher frequencies as in Fig. 6.

A schematic of the enlarged air and fuel injection area is shown in Fig. 7a with $\dot{m}_{\mathrm{i}}$ and $\dot{m}_{\mathrm{o}}$ being the mass flow rate (MFR) from the inner and outer swirlers respectively. The MFRs are calculated using $\dot{m}=$ $\int_{\mathcal{S}} \bar{\rho}\left(\tilde{\boldsymbol{u}} \cdot \boldsymbol{n}_{h}\right) d \mathcal{S}$, where $\boldsymbol{n}_{h}$ is the unit normal vector of the cross-sectional surface, $\mathcal{S}$, which is chosen to be the $h=-8 \mathrm{~mm}$ plane to avoid the density variation close to the flame at $h=0 \mathrm{~mm}$. Different upstream axial locations were also investigated showing similar results.
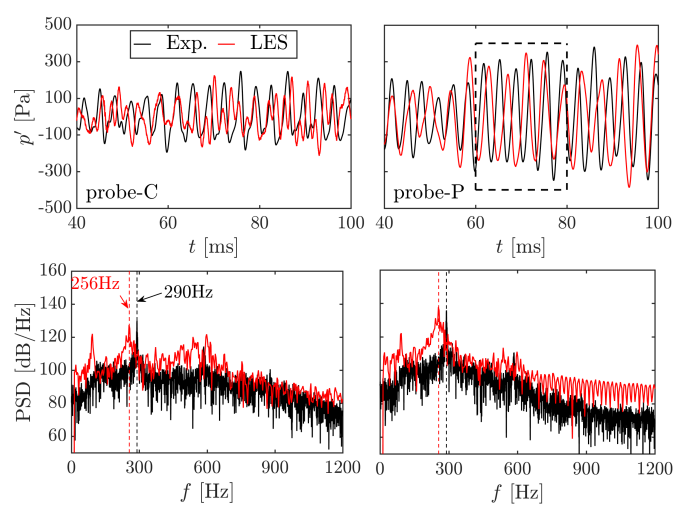

Fig. 6. Pressure fluctuations at probes $\mathrm{C}$ and $\mathrm{P}$ (marked in Fig. 1b) for the unstable flame F-B: time series (top) and PSD (bottom).

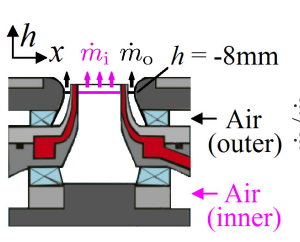

(a)

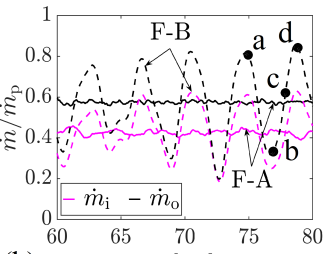

(b)
Fig. 7. Temporal evolution (frame marked in Fig. 6) of air mass flow rates, normalised by $\dot{m}_{\mathrm{p}}$, exiting the inner $\left(\dot{m}_{\mathrm{i}}\right)$ and outer $\left(\dot{m}_{\mathrm{o}}\right)$ swirlers.

In the absence of thermoacoustic instability, the air inflow splits into the two swirlers depending on the hydrodynamic resistance of the flow passages and the MFR ratio between the outer and inner swirler, $\mathcal{R}=$ $\dot{m}_{\mathrm{o}} / \dot{m}_{\mathrm{i}}$, is almost constant for the stable flame F-A, about 1.5 as shown in Fig. 7b. The time window is highlighted in Fig. 6 (dashed box) and $\dot{m}_{\mathrm{p}}$ is the total air MFR injected into the plenum, see Table 1. Both $\dot{m}_{\mathrm{o}}$ and $\dot{m}_{\mathrm{i}}$ vary with time for the flame F-B and the maximum variation is about $50 \%$ and $70 \%$, respectively. This difference in flow response to the pressure oscillation is due to the different acoustic impedances felt by the outer and inner swirlers and as a result, $\mathcal{R}$ varies significantly by about $20 \%$ within a cycle. This leads to a periodic change in the mixing pattern at the combustion chamber inlet.

To elucidate this effect, the time sequence of midplane mass flux magnitude, $\mathcal{M}$, heat release rate, $q$, and mixture fraction contours are shown in Fig. 8 for four typical instances marked in Fig. 7b. The distribution of $q$ is plotted for $|x|>15 \mathrm{~mm}$ to visualise the particular heat release in the chamber outer region due to the flattened flame and an enlarged view of $Z$ contours is shown for $h<10 \mathrm{~mm}$ to identify the upstream mixing patterns. The $x h$-plane mass flux magnitude is computed 
as $\mathcal{M}=\sqrt{(\bar{\rho} \widetilde{u})^{2}+(\bar{\rho} \widetilde{v})^{2}}$, where $\widetilde{u}$ and $\widetilde{v}$ are the filtered axial and radial velocities. The mass fluxes coming through the inner and outer air nozzles are denoted as $\mathcal{M}_{\mathrm{i}}$ and $\mathcal{M}_{\mathrm{o}}$, respectively. Here it is worth noting that the outer nozzle has a conical shape (see Fig. 7a) and thus $\mathcal{M}_{\mathrm{o}}$ tends to move towards the centre, i.e., $x=0$, after exiting the nozzle. By contrast, the inner nozzle is cylindrical and thus $\mathcal{M}_{\mathrm{i}}$ expands outwardly due to the swirl as this flow exits the nozzle.
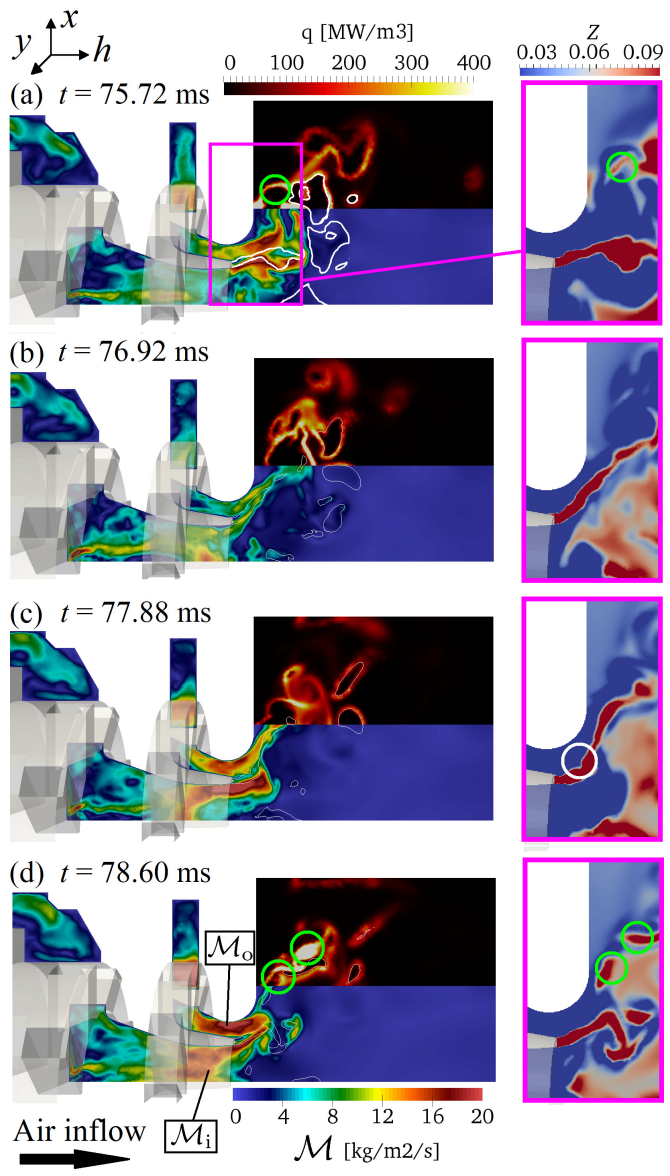

Fig. 8. Time sequence of mid-plane mass flux, heat release rate and mixture fraction contours within one oscillation cycle.

It is seen in Fig. 8a that at instant $t_{a}$ when the ratio $\mathcal{R}$ is at maximum (see Fig. 7b) implying that $\mathcal{M}_{\mathrm{o}}>\mathcal{M}_{\mathrm{i}}$, the fuel jet (red stripe in the enlarged view) is kept quite straight, i.e., $\mathcal{M}_{\mathrm{o}}$ and $\mathcal{M}_{\mathrm{i}}$ are well balanced. This is the mixing pattern observed in F-A (not shown) and all the fuel is consumed in the shear layer between the IRZ and main inflow stream under this condition $[16,17]$. Interestingly, few fuel islands with strong heat release (highlighted by green circles) are seen close to the chamber bottom in the outer region, which is reminiscence of the previous cycle as one shall see later. The ratio $\mathcal{R}$ then decreases with the inner inflow becoming relatively stronger. Half a cycle later at $t_{b}, \mathcal{R}$ reaches its minimum (about 1) and during this process the fuel jet is stretched towards the outer regions as shown in Fig. 8b. Moving to $t_{c}$, the outer-to-inner mass ratio becomes large again and the strong outer flow hits the stretched fuel jet creating an opposite curvature (marked using a white circle in Fig. 8c). Finally in Fig. 8d, the jet breaks down and the main stream recovers the shape shown in Fig. 8a, and then a new cycle begins. The remaining fuel islands are transported towards ORZ where they are eventually consumed due to hot recirculated products. This provides a physical explanation of the high fuel mass fraction for $|x|>15 \mathrm{~mm}$ at $h=5 \mathrm{~mm}$ observed in Fig. 5b, which results in flattening the flame. Moreover, the convective time of these fuel islands are quite different compared to the fuel consumed close to the IRZ, creating a time-lag in the heat release rate which may contribute to the thermoacoustic oscillation forming a feedback sub-system. However, further analysis is required to understand and identify the triggering mechanism, which will be addressed in future investigations. Nevertheless, this study shows that the mass split between the two swirlers arising from the difference in acoustic impedance of nozzle geometries plays an important role in swirl-stabilised flames involving multiple air or fuel injectors. This difference in acoustic response to pressure oscillation introduces periodic variations in mixing, heat release rate and their interactions which can further amplify the combustion oscillations.

\section{Conclusions}

Self-excited thermoacoustic instability and its interaction with fuel-air mixing in a dual swirl GTMC are investigated using LES. Acoustically stable and unstable partially premixed flames are simulated showing good agreement with the measurements for both cases. The oscillation frequency of $290 \mathrm{~Hz}$ for the unstable flame is captured with an under-prediction of $34 \mathrm{~Hz}$ and the computed magnitude agrees quite well with the measurements. The under-prediction of frequency is possibly due to the absence of the fuel plenum in the LES domain and this effect will be investigated in a future study. Based on the good agreement with experiments, the simulation results are then used to obtain new detailed insights into the thermoacoustic interactions with a focus on the previously unexplained transition of flame shape from $\mathrm{V}$-formed to flat when the flames become acoustically unstable. The results show that this change of flame shape, which is well reproduced by the simulation, is closely linked to differences 
in the dynamics of fuel-air mixing for the two flames. The key point is the dynamics of the fuel jets injected between the two swirling air flows. The analysis of air mass flow rate split between the two swirlers reveals that the ratio of these mass flow rates varies significantly during a cycle due to different acoustic impedances of the swirlers. This introduces a periodic radial flapping of the fuel jets, which is inaccessible in experiments, and causes an enhanced radial mixing of fuel that leads to the shorter and flattened flame shape found in the unstable case.

\section{Acknowledgements}

ZXC and NS acknowledge the support of Mitsubishi Heavy Industries, Japan. This work used the ARCHER UK National Supercomputing Service (http://www.archer.ac.uk) with the computational time provided by the UKCTRF.

\section{References}

[1] S. Candel, Proc. Combust. Inst. 29 (2002) 1-28.

[2] T.C. Lieuwen, V. Yang (Eds.), Combustion instabilities in gas turbine engines: Operational experience, fundamental mechanisms, and modeling, AIAA Inc., Reston, VA, 2006.

[3] T. Poinsot, D. Veynante, Theoretical and Numerical Combustion, Edwards, second edition, 2005.

[4] A.P. Dowling, J. Fluid Mech. 346 (1997) 271-290.

[5] N. Noiray, D. Durox, T. Schuller, S. Candel, J. Fluid Mech. 615 (2008) 139-167.

[6] T.C. Lieuwen, J. Propul. Power 19 (2003) 765-781.

[7] A.P. Dowling, S.R. Stow, J. Propul. Power 19 (2003) 751-764.

[8] C.F. Silva, F. Nicoud, T. Schuller, D. Durox, S. Candel, Combust. Flame 160 (2013) 1743-1754.

[9] T. Poinsot, Proc. Combust. Inst. 36 (2017) 1-28.

[10] S. Roux, G. Lartigue, T. Poinsot, U. Meier, C. Bérat, Combust. Flame 141 (2005) 40-54.

[11] B. Franzelli, E. Riber, L.Y.M. Gicquel, T. Poinsot, Combust. Flame 159 (2012) 621-637.

[12] J.-M. Lourier, M. Stöhr, B. Noll, B. Werner, A. Fiolitakis, Combust. Flame 183 (2017) 343-357.

[13] G. Staffelbach, L.Y.M. Gicquel, G. Boudier, T. Poinsot, Combust. Flame 32 (2009) 2909-2916.

[14] P. Wolf, G. Staffelbach, L.Y.M. Gicquel, J.-D. Müller, T. Poinsot, Combust. Flame 159 (2012) 3398-3413.

[15] Y.M. Al-Abdeli, A.R. Masri, Exp. Therm. Fluid Sci. 69 (2015) 178-196.

[16] P. Weigand, W. Meier, X.R. Duan, W. Stricker, M. Aigner, Combust. Flame 144 (2006) 205-224.

[17] W. Meier, X.R. Duan, P. Weigand, Combust. Flame 144 (2006) 225-236.

[18] M. Stöhr, C.M. Arndt, W. Meier, Proc. Combust. Inst. 34 (2013) 3107-3115.

[19] Y.C. See, M. Ihme, Proc. Combust. Inst. 35 (2015) 1225-1234.

[20] A. Donini, R.J.M. Bastiaans, J.A. van Oijen, L.P.H. de Goey, Flow Turbul. Combust. 98 (2017) 887-922.

[21] A.C. Benim, S. Iqbal, W. Meier, F. Joos, A. Wiedermann, Appl. Therm. Eng. 110 (2017) 202-212.
[22] Z. Chen, S. Ruan, N. Swaminathan, Proc. Combust. Inst. 36 (2017) 1645-1652.

[23] S. Ruan, N. Swaminathan, O.R. Darbyshire, Combust. Theory Model. 18 (2014) 295-329.

[24] Z. Chen, S. Ruan, N. Swaminathan, Combust. Flame 162 (2015) 703-716.

[25] S. Ruan, N. Swaminathan, M. Isono, T. Saitoh, K Saitoh, J. Propul. Power 31 (2015) 861-871.

[26] A. Widenhorn, B. Noll, M. Aigner, AIAA Paper 647 (2009).

[27] M. Stöhr, Z. Yin, W. Meier, Proc. Combust. Inst. 36 (2017) 3907-3915. 